\title{
Reliability of the time splitting Fourier method for singular solutions in quantum fluids
}

\author{
M. Caliari ${ }^{\mathrm{a}, *}$, S. Zuccher ${ }^{\mathrm{a}}$ \\ ${ }^{a}$ Department of Computer Science, University of Verona, Strada Le Grazie 15, \\ 37134 Verona, Italy
}

\begin{abstract}
We extensively study the numerical accuracy of the well-known time splitting Fourier spectral method for the approximation of singular solutions of the GrossPitaevskii equation. In particular, we explore its capability of preserving a steady-state vortex solution, whose density profile is approximated by a very accurate diagonal Padé expansion of order 8 , here explicitly derived for the first time. Although the Fourier spectral method turns out to be only slightly more accurate than a time splitting finite difference scheme, the former is reliable and efficient. Moreover, at a post-processing stage, it allows an accurate evaluation of the solution outside grid points, thus becoming particularly appealing when high resolution is needed, such as in the study of quantum vortex interactions.
\end{abstract}

Keywords: Quantum fluids, nonuniform finite differences, time splitting, Fourier spectral method

\section{Introduction}

Quantum turbulence [1 3], as well as classical turbulence [4, 5], is dominated by reconnection of vortical structures which is much simpler to treat in the framework of quantum fluids rather than in viscous fluids [6], while leading to similar features such as time asymmetry [7]. Despite the fundamental differences between the two forms of turbulence, there are reasons to believe that the understanding of quantum turbulence might shed new light on the understanding of its classical counterpart [3].

Quantum fluids dynamics is properly described by the Gross-Pitaevskii equation (GPE) [8, 9]

$$
\frac{\partial \psi}{\partial t}=\frac{\mathrm{i}}{2} \nabla^{2} \psi+\frac{\mathrm{i}}{2}\left(1-|\psi|^{2}\right) \psi
$$

where $\psi$ is the complex wave function. Through the Madelung transformation $\psi=\sqrt{\rho} \exp (\mathrm{i} \theta)$, equation (11) can be viewed in classical fluid dynamical terms

${ }^{*}$ Corresponding author 


$$
\begin{aligned}
\frac{\partial \rho}{\partial t}+\frac{\partial\left(\rho u_{j}\right)}{\partial x_{j}} & =0 \\
\rho\left(\frac{\partial u_{i}}{\partial t}+u_{j} \frac{\partial u_{i}}{\partial u_{j}}\right) & =-\frac{\partial p}{\partial x_{i}}+\frac{\partial \tau_{i j}}{\partial x_{j}},
\end{aligned}
$$

where $\rho=|\psi|^{2}$ denotes density, $\boldsymbol{u}=\boldsymbol{\nabla} \theta$ velocity, $p=\frac{\rho^{2}}{4}$ pressure, and $\tau_{i j}=\frac{1}{4} \rho \frac{\partial^{2} \ln \rho}{\partial x_{i} \partial x_{j}}$ the so-called quantum stress $(i, j=1,2,3)$. Defects in the wave function $\psi$ are interpreted as infinitesimally thin vortices of constant circulation $\Gamma=\oint \boldsymbol{u} \cdot \mathrm{d} \boldsymbol{s}=2 \pi$, with healing length $\xi=1$. GPE conserves the mass and the energy

$$
E=\frac{1}{2} \int|\nabla \psi|^{2} \mathrm{~d} x+\frac{1}{4} \int\left(1-|\psi|^{2}\right)^{2} \mathrm{~d} x .
$$

The main reason for preferring the GPE approach to others for the study of quantum turbulence is that it guarantees a natural dynamics of interacting vortices [10] while resolving fine scales up to the vortex core [3, 11]. On the contrary, methods based on the inviscid Euler equations (either their direct numerical simulation [12] or vortex filament methods [13]) are unable to automatically perform vortex reconnections, being forbidden by Euler dynamics.

The numerical solution of the GPE (1) is normally carried out by employing time splitting Fourier methods [7, 10, 14, 15] and by imposing vortices in the form of singular phase defects in a unitary background density, i.e. $\rho(x)=$ $|\psi(x)|^{2} \rightarrow 1$ when $|x| \rightarrow \infty$. However, these methods assume periodic boundary conditions. Solutions which are not periodic must be mirrored in the directions lacking periodicity [14], thus imposing doubling of the degrees of freedom in each of those directions and a consequent increase of the computational effort.

Recent developments [10, 16] have shown that reconnections in quantum fluids are strictly related to topological features characterizing the interacting vortex tubes such as writhe, total torsion and intrinsic twist. These quantities depend on the fine details of the curve that describes the vortex centerline (its third derivative with respect to the curvilinear abscissa is required for computing torsion) and on the phase of the wavefunction $\psi$ in the neighborhood of the vortex centerline. Therefore it is paramount to resort to high resolution numerical simulations of equation (1), especially in the proximity of the reconnection event.

With the goal of assessing the goodness of time-splitting Fourier methods for singular solutions on uniform grids versus time-splitting finite differences on nonuniform grids, we first derive an analytic approximation of a two-dimensional steady state vortex that nullifies the right-hand-side of (10). Then we perform a systematic comparison between the two approaches by measuring the deviation of the numerical solution from the initial condition (being steady the initial condition should remain preserved). Finally, we explore the possibility to evaluate the solution obtained by time-splitting Fourier methods on nonuniform 
grids designed to guarantee higher spatial resolution in the proximity of vortex singularities.

\section{Accurate Padé approximation of a $2 d$ vortex}

We seek a two-dimensional, steady-state solution of equation (1) that represents a straight vortex centered at the origin. It is well-known that the classical two-dimensional Euler vortex of circulation $\Gamma$ has azimuthal velocity $u_{\theta}=\Gamma /(2 \pi r)$ where $r=\sqrt{x_{1}^{2}+x_{2}^{2}}$ is the radius and $\theta=\operatorname{atan} 2\left(x_{2}, x_{1}\right)=$ $\arg \left(x_{1}+\mathrm{i} x_{2}\right) \in(-\pi, \pi]$ is the azimuthal angle. The Cartesian components of the velocity are thus $u_{1}=-u_{\theta} \sin \theta=-\Gamma x_{2} /\left(2 \pi r^{2}\right)$ and $u_{2}=u_{\theta} \cos \theta=\Gamma x_{1} / r^{2}$. Therefore $\mathbf{u}=\left(u_{1}, u_{2}\right)=(\Gamma /(2 \pi)) \boldsymbol{\nabla} \theta$. This shows that the velocity field is solenoidal $(\boldsymbol{\nabla} \cdot \mathbf{u}=0)$, that the quantum mechanical phase, $S$, is simply the azimuthal angle $\theta$, and that the quantum of circulation, in our dimensionless units, is equal to $2 \pi$. In steady conditions, the continuity equation ensures that $\boldsymbol{\nabla} \cdot(\rho \mathbf{u})=0$, hence $\mathbf{u} \cdot \boldsymbol{\nabla} \rho=0$, which means that $\boldsymbol{\nabla} \rho \cdot \boldsymbol{\nabla} \theta=0$. The solution $\rho=\bar{\rho}=$ const leads to $\psi=\sqrt{\bar{\rho}} \mathrm{e}^{\mathrm{i} \theta}$, which has infinite energy (44) and must be rejected. The other possibility is that $\nabla \rho \perp \nabla \theta$. Since $\nabla \theta=\hat{\theta} / r$, then $\nabla \rho$ is parallel to $\hat{r}$ and thus $\rho=\rho(r), \hat{r}$ and $\hat{\theta}$ being the unitary vectors in two-dimensional polar coordinates.

In a two-dimensional domain we set $\psi\left(x_{1}, x_{2}\right)=\rho\left(\sqrt{x_{1}^{2}+x_{2}^{2}}\right)^{1 / 2} \mathrm{e}^{\mathrm{i} \theta\left(x_{1}, x_{2}\right)}=$ $f\left(\sqrt{x_{1}^{2}+x_{2}^{2}}\right) \mathrm{e}^{\mathrm{i} \theta\left(x_{1}, x_{2}\right)}$, where $f\left(\sqrt{x_{1}^{2}+x_{2}^{2}}\right)=f(r)$ is a function to be determined. By imposing that $\psi$ is the steady solution of equation (11), we find that $f$ satisfies the equation

$$
f^{\prime \prime}+\frac{f^{\prime}}{r}+f\left(1-f^{2}-\frac{1}{r^{2}}\right)=0,
$$

with boundary conditions $f(0)=0, f(\infty)=1$.

Equation (5) could be integrated numerically as it is, by artificially bounding the infinite domain. To avoid this problem, we resort to the change of variables $s=r /(1+r), g(s)=f(r)$, which yields the equation for $g(s)$

$$
(s-1)^{4} g^{\prime \prime}+2(s-1)^{3} g^{\prime}-\frac{(s-1)^{3}}{s} g^{\prime}-\frac{(s-1)^{2}}{s^{2}} g+\left(1-g^{2}\right) g=0,
$$

defined in the finite domain $s \in(0,1]$, with boundary conditions $g(0)=0$ and $g(1)=1$. We solve equation (6) by central second order finite differences with equally spaced discretization points $s_{i}=i / N, i=1,2, \ldots, N$. Given the numerical solution $\tilde{g}$ of (6), the numerical approximation of the density is

$$
\rho_{\text {num }}\left(r_{i}\right)=\left[\tilde{g}\left(\frac{r_{i}}{1+r_{i}}\right)\right]^{2}
$$

where $r_{i}=s_{i} /\left(1-s_{i}\right), i=1,2, \ldots, N-1$. This rescaling provides denser points $r_{i}$ in the neighborhood of the origin, where they are mostly needed (more than 
$95 \%$ of the points $r_{i}$ are in the interval $0 \leq r \leq 20$ ). Nevertheless, the computation of the initial solution for (11) on a two-dimensional grid, for instance, requires $\rho_{\text {num }}$ to be interpolated.

It would be therefore useful to have an analytic approximation of $f(r)$. However, since it is more convenient [17] to find a Padé approximation directly for $\rho(r)=[f(r)]^{2}$ rather than for $f(r)$, we rewrite equation (5) in terms of $\rho(r)$ as

$$
\rho^{\prime \prime}+\frac{\rho^{\prime}}{r}-\frac{\left(\rho^{\prime}\right)^{2}}{2 \rho}-\frac{2 \rho}{r^{2}}+2(1-\rho) \rho=0,
$$

with boundary conditions $\rho(0)=0, \rho(\infty)=1$.

It is known [17, 18] that Padé approximations of $\rho(r)$ retain only even degrees at both the numerator and denominator, that is

$$
\rho(r) \approx \frac{\sum_{j=0}^{p} a_{j} r^{2 j}}{1+\sum_{k=1}^{q} b_{k} r^{2 k}}=\frac{a_{0}+a_{1} r^{2}+a_{2} r^{4}+\cdots+a_{p} r^{2 p}}{1+b_{1} r^{2}+b_{2} r^{4}+\cdots+b_{q} r^{2 q}} .
$$

In order for this approximation to satisfy the boundary conditions, it must be

$$
\begin{aligned}
\rho(0) & =0 \\
\rho(\infty) & \Longrightarrow 1 \quad \Longrightarrow a_{0}=0 \\
& \Longrightarrow p=q, \quad b_{q}=a_{p} .
\end{aligned}
$$

Given these simplifications, the diagonal Padé approximation, with $2 q-1$ coefficients and both numerator and denominator of degree $r^{2 q}$, is

$$
\rho_{q}(r)=\frac{a_{1} r^{2}+a_{2} r^{4}+a_{3} r^{6}+\cdots+a_{q} r^{2 q}}{1+b_{1} r^{2}+b_{2} r^{4}+b_{3} r^{6}+\cdots+a_{q} r^{2 q}} .
$$

In literature this approximation is normally limited to $q=2$ [17], that is

$$
\rho_{2}(r)=\frac{a_{1} r^{2}+a_{2} r^{4}}{1+b_{1} r^{2}+a_{2} r^{4}}
$$

with

$$
a_{1}=\frac{11}{32}, \quad b_{1}=\frac{5-32 a_{1}}{48-192 a_{1}}, \quad a_{2}=a_{1}\left(b_{1}-\frac{1}{4}\right) .
$$

Despite its widespread usage, this approximation is qualitatively wrong (see, e.g., [19]), because it reaches an unphysical maximum, above $\rho(\infty)=1$, at $r_{0}=2 \sqrt{6(4+3 \sqrt{2})} \approx 14.065$, unique positive solution of $r^{4}-192 r^{2}-1152$ obtained by imposing $\rho_{2}^{\prime}(r)=0$. The physical solution of equation (8) must reach the limit value $\rho(\infty)=1$ monotonically, without overshooting.

Due to these limitations, we seek higher-order $(q>2)$, monotonically increasing, Padé expansions, namely

$$
\rho_{3}(r)=\frac{a_{1} r^{2}+a_{2} r^{4}+a_{3} r^{6}}{1+b_{1} r^{2}+b_{2} r^{4}+a_{3} r^{6}} \quad \text { and } \quad \rho_{4}(r)=\frac{a_{1} r^{2}+a_{2} r^{4}+a_{3} r^{6}+a_{4} r^{8}}{1+b_{1} r^{2}+b_{2} r^{4}+b_{3} r^{6}+a_{4} r^{8}} .
$$


In order to determine the coefficients of a certain approximation $\rho_{q}(r)$, we compute the analytic expressions $\rho_{q}(r), \rho_{q}^{\prime}(r)$ and $\rho_{q}^{\prime \prime}(r)$ and substitute them in equation (8) obtaining the form

$$
\rho_{q}^{\prime \prime}+\frac{\rho_{q}^{\prime}}{r}-\frac{\left(\rho_{q}^{\prime}\right)^{2}}{2 \rho_{q}}-\frac{2 \rho_{q}}{r^{2}}+2\left(1-\rho_{q}\right) \rho_{q}=0 \Longleftrightarrow \frac{N_{q}(r)}{D_{q}(r)}=0 .
$$

The numerator $N_{q}(r)$ is made of terms $r^{2 k}$, which are in a number much larger than the $2 q-1$ degrees of freedom of the Padé expansion. For this reason equation (8) cannot be satisfied exactly. However, we can nullify the coefficients of $2 q-1$ terms $r^{2 k}$. We can choose to start from higher- or lower-order coefficients in $N_{q}(r)$. We prefer to operate on lower-order powers of $r^{2 k}$, i.e. $k=1, \ldots, 2 q-1$, because we need a good approximation of $\rho_{q}(r)$ in a neighborhood of the origin. Interestingly enough, we observe a posteriori that canceling the lower-order coefficients of $r^{2 k}$ results in very small values of the coefficients of larger powers of $r$. The step-by-step derivation of $\rho_{q}(r)$ for $q=2,3,4$ is reported in Appendix A, whereas tables A.1 and A.2 summarize all coefficients for the expansions $\rho_{q}, q=2,3,4$.

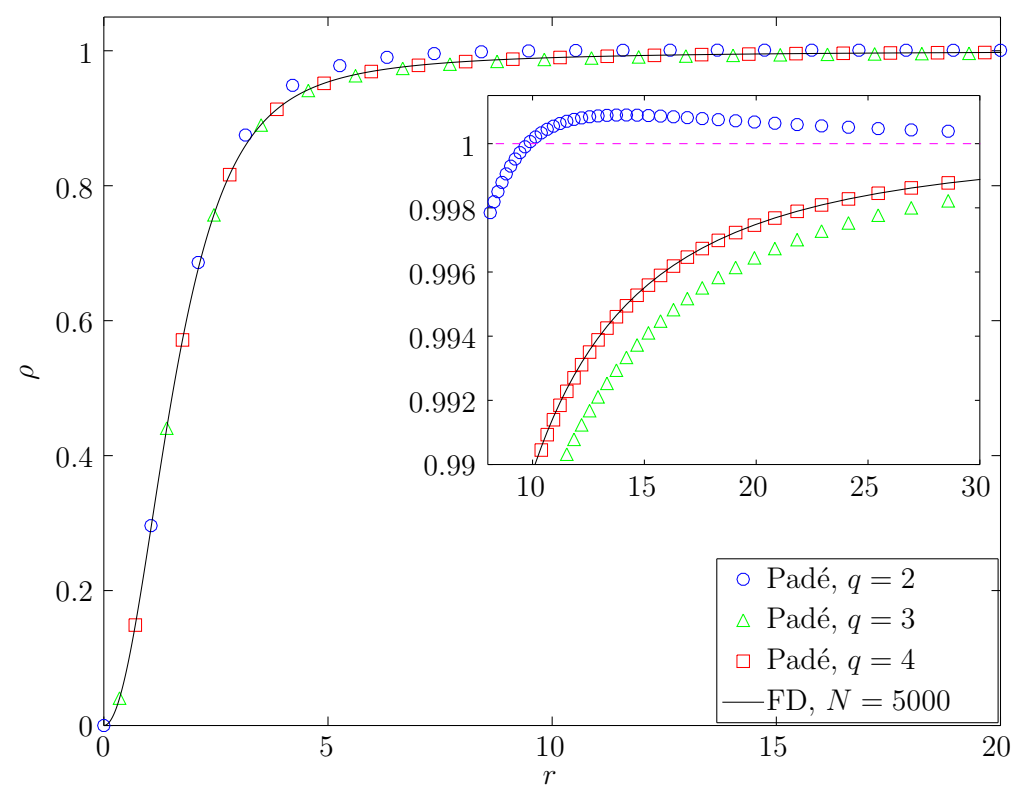

Figure 1: Comparison between $\left[\tilde{g}\left(\frac{r}{1+r}\right)\right]^{2}$, numerical solution of [6] obtained by secondorder finite differences on 5000 equispaced points, and different Padé approximations $\rho_{q}(r)$.

In Figure1 we show the comparison between the numerical solution of (6) by employing second-order central finite differences with 5000 points and different 
Padé approximations $\rho_{q}(r)$ for $q=2,3,4$. Visual inspection confirms that $q=2$ is a poor representation of the solution of equation (8), especially for $4<r<20$.

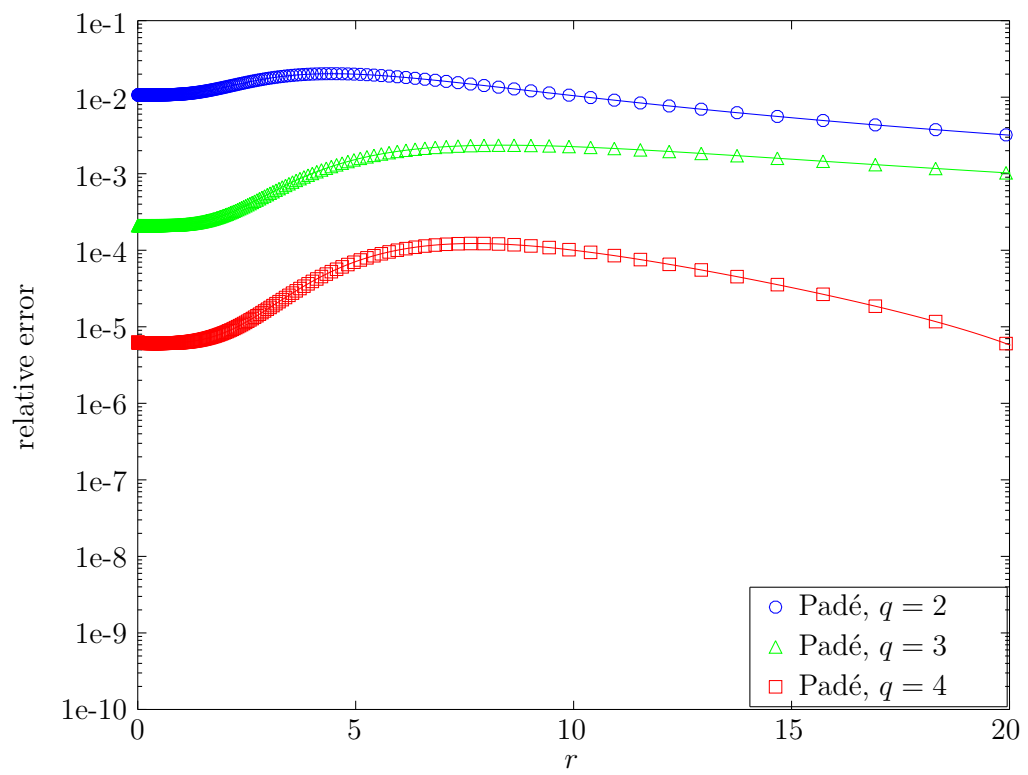

Figure 2: Relative error between different Padé approximations $\rho_{q}(r)$ and the numerical solution of (6) with (equally spaced grid, second-order finite differences, 5000 points).

To appreciate quantitatively the error with respect to the numerical solution, in Figure 2 we report the relative error in a semilog plot. Interestingly, the relative error does not reach its maximum close to the origin, meaning that any Padé approximation reproduces quite well the behavior of the vortex for $r \rightarrow 0$. On the other hand, the maximum relative error is always reached for $r<10$, i.e. in a region of interest for the numerical simulations that we will perform.

\section{Time splitting methods}

Widely used schemes for the numerical simulation of the dynamics of (1D) are the so-called time-splitting methods and the finite difference time domain methods (see [20] for a review). If we restrict the options to second-order accurate schemes in time, Time Splitting pseudoSPectral (TSSP) methods, Time Splitting Finite Difference (TSFD) methods and Crank-Nicolson Finite Difference (CNFD) method conserve the mass at the discretized level. However, CNFD is implicit and requires the solution of a coupled nonlinear system at each time step. For this reason we resorted to time splitting methods. We refer the reader to 21] for higher-order time splitting methods. 
In 20, Example 4.1] TSSP is suggested when the solution is smooth and TSFD otherwise, although the hint comes from a one-dimensional numerical experiment. In what follows, we analyze two approaches: a classical time splitting Fourier method and a time splitting nonuniform finite difference method. In any case, equation (11) is split into two parts

$$
\begin{aligned}
& \frac{\partial u}{\partial t}(t, x)=\frac{\mathrm{i}}{2} \nabla^{2} u(t, x) \\
& \frac{\partial v}{\partial t}(t, x)=\frac{\mathrm{i}}{2}\left(1-|v(t, x)|^{2}\right) v(t, x)
\end{aligned}
$$

where $x=\left(x_{1}, \ldots, x_{d}\right) \in \mathbb{R}^{d}$. The solution of the first equation depends on the space chosen for the discretization and will be described in the next two sections. The second equation can be solved exactly, taking into account that $|v|$ is preserved by the equation. Therefore

$$
v(\tau, x)=\exp \left(\frac{\tau \mathrm{i}}{2}\left(1-|v(0, x)|^{2}\right)\right) v(0, x)
$$

for any $x$ in the spatial domain. If we denote by $\mathrm{e}^{\tau \mathcal{A}} u_{n}(x)$ and $\mathrm{e}^{\tau \mathcal{B}\left(v_{n}(x)\right)} v_{n}(x)$ the two partial numerical solutions, the approximation $\psi_{n+1}(x)$ of $\psi\left(t_{n+1}, x\right)$, where $t_{n+1}=(n+1) \tau$, can be recovered by the so-called Strang splitting

$$
\begin{aligned}
\psi_{n+1 / 2}(x) & =\mathrm{e}^{\tau \mathcal{A}} \mathrm{e}^{\frac{\tau}{2} \mathcal{B}\left(\psi_{n}(x)\right)} \psi_{n}(x) \\
\psi_{n+1}(x) & =\mathrm{e}^{\frac{\tau}{2} \mathcal{B}\left(\psi_{n+1 / 2}(x)\right)} \psi_{n+1 / 2}(x) .
\end{aligned}
$$

\subsection{Time splitting Fourier method}

Equation (12a) can be solved exactly in time within the Fourier spectral space. A part from the error at machine-precision level coming from the necessary direct and inverse Fast Fourier Transforms (FFTs), the only possible considerable error might arise from an insufficient number of Fourier modes. This is usually not a big deal when approximating smooth solutions fastly decaying to zero, since spectral order of convergence takes place. For this to happen, the unbounded domain has to be truncated to a computational bounded domain $\Omega$ large enough to support the most of a periodic approximation of the solution. However, when simulating the dynamics of vortex solutions not decaying to zero, as in our case where $\lim _{|x| \rightarrow \infty}|\psi(t, x)|=1$, there are some issues to take into account: the low regularity of the solution at the origin, due both to $\rho(t, 0)$ and to $\theta(t, 0)$, and lack of periodicity at the boundaries, also considering the usual extension of the computational domain and reflection of the solution (see [14]). In fact, after such a mirroring, the solution takes the same values at opposite boundaries, but its derivatives do not.

In order to investigate the accuracy of Fourier approximation for vortex solutions, we consider the Fourier series expansion of the function obtained by mirroring 


$$
\psi_{0}(r, \theta)=\left(1-\left(1-\rho_{4}^{q / 2}(r)\right) \mathrm{e}^{-r^{2} / \ell^{2}}\right) \mathrm{e}^{c \mathrm{i} \theta \mathrm{e}^{-r^{2} / \ell^{2}}}, \quad \psi_{0}:[-L, L)^{2} \rightarrow \mathbb{C}
$$

with respect to the axis $x=L$ and $y=L$. The final computational domain is therefore $\Omega=[-L, 3 L)^{2}$, with $L=20$, for which $\rho_{4}(L, L) \approx 0.99875$. The choice of the parameters $q=1, \ell \rightarrow \infty$ and $c=1$ corresponds to a twodimensional straight vortex as described above. Different choices provide more regular functions or functions fastly decaying to zero, for which the derivatives at the boundaries are almost periodic. We compute a reference approximation by an expansion into a series with $2048^{2}$ Fourier modes and compare it with expansions ranging between $M=m^{2}=16^{2}$ and $M=m^{2}=512^{2}$ modes, in the $L^{2}$ norm. For a quite regular and periodic function, corresponding to $q=10$,

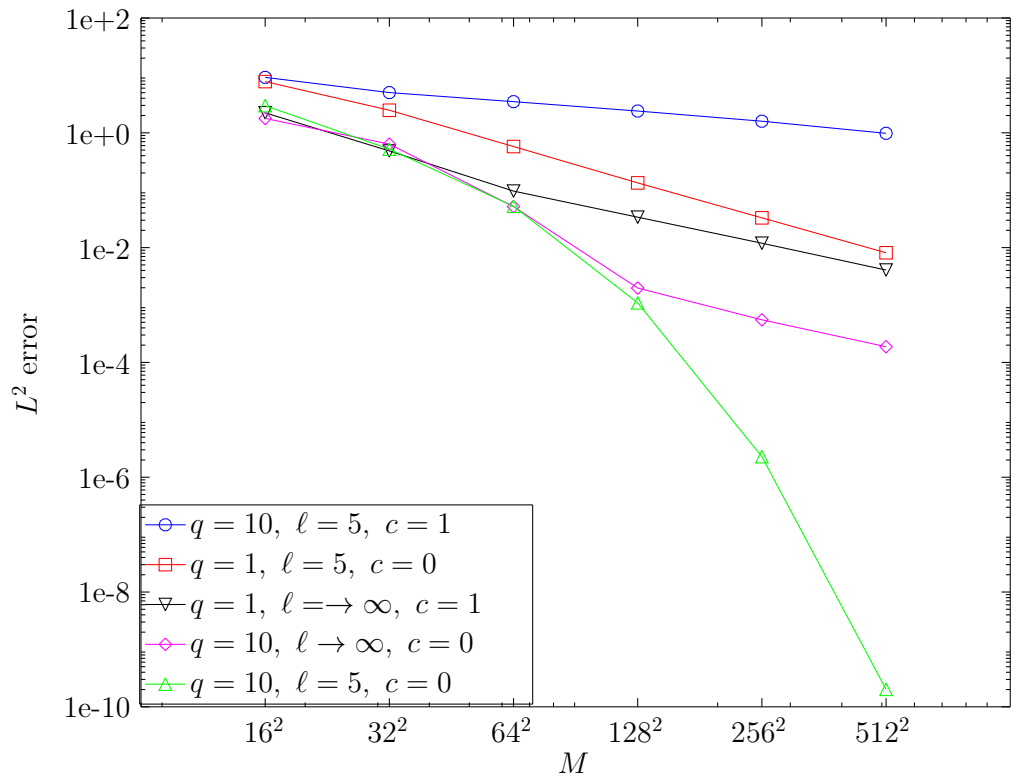

Figure 3: Error behavior of the Fourier approximation of function (14) (extended to $[-L, 3 L)^{2}$ by mirroring) for different choices of the parameters. Only the case of a regular and fast decaying to zero function ( $q=10, \ell=5$ and $c=0$, green, upward triangles) shows the typical spectral rate of convergence.

$\ell=5$ and $c=0$ we observe in Figure 3 a typical spectral rate of convergence. For any other choice of the parameters, which affects the regularity of the density $(q=1, \ell=5, c=0)$, or the fast decay to zero of the function and its derivatives $(q=10, \ell \rightarrow \infty, c=0)$ or the regularity of the phase $(q=10, \ell=5, c=1)$ we observe a strong order reduction. The same reduction occurs for the straight vortex $(q=1, \ell \rightarrow \infty, c=0)$. 
Increasing the number of Fourier coefficients so as to gain accuracy is often not an option. In fact, due to the necessary mirroring, this corresponds to a huge growth of the degrees of freedom. Moreover, the use of hyperbolic sparse grids (see 22], for instance) is not possible, since the possibility of discarding coefficients and grid points is given only for highly regular solutions.

The low regularity of the solutions to be approximated and the needed duplication along axes in order to satisfy at least the periodicity of the values of the solutions suggest to explore the alternative of a finite difference discretization in space.

\subsection{Time splitting finite difference method}

The main advantage of a finite difference approach is that the mirroring of the solution is not required, being the extension of the bounded domain replaced by the imposition of homogeneous Neumann boundary conditions.

Given the low regularity of vortex solutions, we use centered second order finite differences. With the aim of increasing the spatial resolution around the vortex cores and keeping a reasonable degree of freedom, we employ a set of nonuniform grid points (see [23], for instance, for locally adaptive finite element discretizations).

The discretization of the Laplace operator in one dimension with nonuniform finite differences on $m$ points provides the nonsymmetric matrix

$$
A_{1}=\left[\begin{array}{ccccc}
-\frac{2}{h_{1}^{2}} & \frac{2}{h_{1}^{2}} & 0 & \cdots & 0 \\
\frac{2}{h_{1}\left(h_{1}+h_{2}\right)} & -\frac{2}{h_{1} h_{2}} & \frac{2}{h_{2}\left(h_{1}+h_{2}\right)} & \ddots & 0 \\
0 & \ddots & \ddots & \ddots & 0 \\
0 & \ddots & \frac{2}{h_{m-2}\left(h_{m-2}+h_{m-1}\right)} & -\frac{2}{h_{m-2} h_{m-1}} & \frac{2}{h_{m-1}\left(h_{m-2}+h_{m-1}\right)} \\
0 & \cdots & 0 & \frac{2}{h_{m-1}^{2}} & -\frac{2}{h_{m-1}^{2}}
\end{array}\right]
$$

where $h_{i}=x_{i+1}-x_{i}, x_{1}=-L, x_{m}=L$. This is not exactly a second order approximation, although a discretization in which $h_{i+1}=(1+\delta) h_{i}$ and a refinement with $h_{j+1}=(1+\delta)^{1 / 2} h_{j}$ yields a first order term in the error decaying faster than the second order term (see [24, § 3.3.4]). The approximation for the two-dimensional and the three-dimensional cases can be simply obtained by Kronecker products with the identity matrix. If we call $A$ the corresponding matrix, equation (12a) is transformed into the system of ordinary differential equations

$$
y^{\prime}(t)=\frac{\mathrm{i}}{2} A y(t), \quad y(t) \in \mathbb{C}^{M \times 1} .
$$

Given the importance of the mass preservation, we investigate this issue for the numerical solution of system (15). A quadrature formula with positive weights for the computation of the mass writes

$$
\int_{\Omega}|\psi(t, x)|^{2} \mathrm{~d} x \approx w^{T}|y(t)|^{2}, \quad w \in \mathbb{R}_{+}^{M \times 1} .
$$


It can be written as

$$
y(t)^{*} W y(t)
$$

where $y(t)^{*} \in \mathbb{C}^{1 \times M}$ denotes the transposed conjugate vector of $y(t)$ and $W$ the matrix with diagonal $w$. We define $z(t)=W^{1 / 2} y(t)$ such that

$$
z^{\prime}(t)=\frac{\mathrm{i}}{2} A_{w} z(t)
$$

with $A_{w}=W^{1 / 2} A W^{-1 / 2}$. If $A_{w}$ is symmetric, then the solution $z(\tau)=$ $\exp \left(\tau \mathrm{i} / 2 A_{w}\right)$ is an orthogonal matrix and

$$
z(\tau)^{*} z(\tau)=z^{*}(0) z(0) .
$$

This means that

$$
\begin{aligned}
y(\tau)^{*} W y(\tau) & =\left(W^{1 / 2} y(\tau)\right)^{*}\left(W^{1 / 2} y(\tau)\right)=z(\tau)^{*} z(\tau)=z(0)^{*} z(0)= \\
& =\left(W^{1 / 2} y(0)\right)^{*}\left(W^{1 / 2} y(0)\right)=y(0)^{*} W y(0)
\end{aligned}
$$

and therefore system (15) preserves the mass at the discrete level if $W$ makes $A_{w}$ symmetric. From the structure of the matrix $A_{1}$, it is clear that the vector of trapezoidal weights $w^{T}=\left[h_{1}, h_{1}+h_{2}, h_{2}+h_{3}, \ldots, h_{m-1}\right]$ gives a matrix $W_{1}$ such that $W_{1} A_{1}$ is symmetric. The extension to $W$ in the two-dimensional and three-dimensional cases is trivial and this is enough to get $A_{w}$ symmetric as well, in fact $A^{T} W=W A \Longleftrightarrow W^{-1 / 2} A^{T} W=W^{1 / 2} A \Longleftrightarrow W^{-1 / 2} A^{T} W^{1 / 2}=$ $A_{w}^{T}=W^{1 / 2} A W^{-1 / 2}=A_{w}$. We conclude that equation (15) preserves the mass at the discrete level whenever the trapezoidal rule is used as quadrature formula and this is easily extended to any space dimension.

System (16) could be solved, for instance, by the Crank-Nicolson scheme

$$
z_{n+1}=z_{n}+\frac{k \mathrm{i}}{4} A_{w} z_{n}+\frac{k \mathrm{i}}{4} A_{w} z_{n+1},
$$

which preserves the discrete mass being $A_{w}$ symmetric (see [20]). This scheme is second order accurate in time, therefore the size of the time step $k$ has to be chosen such that the error is smaller than the time splitting error. Moreover, Crank-Nicolson scheme requires the solution of a linear system of equations with matrix $\left(I-k \mathrm{i} A_{w} / 4\right)$ at each time step $k$. Although this is not a big deal in one space dimension, since the matrix is tridiagonal, in higher dimensions the discretization yields a large, sparse, complex symmetric matrix. This implies the use of preconditioned Krylov solvers for general matrices such as GMRES or BiCGStab or minimal residual methods for complex symmetric systems (see 25]). Iterative methods converge to the solution up to a specified tolerance which therefore influences the mass conservation and the whole accuracy of the result. Given these complications, we prefer to consider a direct approximation of the exact solution

$$
z_{n+1}=\exp \left(\tau \mathrm{i} / 2 A_{w}\right) z_{n} .
$$

Nowadays there are several options for the computation of the action of the matrix exponential to a vector. We refer to [26] for a review of polynomial 
methods which do not require the solution of linear systems. In this way, the kinetic linear part (12a) is solved exactly in time, as in the Fourier spectral method.

\section{Numerical experiments}

In Section 2 we have derived various approximations of $\rho(r)$ for a straight, two-dimensional vortex, whose wavefunction is $\psi(r, \theta)=\sqrt{\rho} \mathrm{e}^{\mathrm{i} \theta}$. In order to quantitatively compare the two methods introduced in Section 3 , we measure the preservation of such a steady solution by reporting the relative error

$$
\max _{0<|r| \leq R} \frac{\left|\psi_{n}(r, \theta)-\psi_{0}(r, \theta)\right|}{\left|\psi_{0}(r, \theta)\right|}, \quad n=1,2, \ldots, T / \tau
$$

with $\psi_{0}(r, \theta)=\left|\psi_{0}(r)\right| \mathrm{e}^{\mathrm{i} \theta}$, where $\left|\psi_{0}(r)\right|$ is either $\sqrt{\rho_{q}(r)}$ or $\sqrt{\rho_{\text {num }}(r)}$, the latter evaluated at any required $r$ by linear interpolation of (17). The origin is excluded since $\psi_{0}$ is zero therein. The time step $\tau$ is chosen such that $T / \tau$ is an integer, where $T$ is the final simulation time. In all our experiments, we selected $T=10$, a reasonable value in quantum fluids simulations [7, 10, 15]. The maximum over the continuum set $\{0<|r| \leq R\}$ in the error above is approximated by the maximum over a discrete set which will be specified later.

Although the preservation of the initial state may seem a trivial test, it is in fact a reliable and necessary experiment in order to validate the effectiveness of the proposed numerical methods. Thanks to the reliability of the analytic solution, this test can show the influence of both the spatial approximation and the time splitting error in the numerical discretization of the PDE (11).

In what follows we will employ either TSSP (Fourier) or TSFD. For a computational grid with $m \times m$ grid points in the physical domain of interest, TSSP requires a total of $M=2 m \times 2 m=4 m^{2}$ degrees of freedom due to mirroring, whereas TSFD requires only $M=m^{2}$ degrees of freedom thanks to homogeneous Neumann boundary conditions.

\subsection{Comparison between different approximations of the initial condition}

We preliminary test the reliability of the three Padé approximations $\rho_{q}, q=$ $2,3,4$ and the numerical solution of equation (6) obtained by central second order finite differences with $N=5000$ uniformly distributed discretization points. For the solution of the GPE (1) we employ TSSP with Fourier basis functions on a uniform two-dimensional computational grid. For this reason, the numerical solution $\tilde{g}(s)$ of equation (6) must be interpolated.

Results are reported in Figure 4, where the relative error defined by (17) is plotted versus time for different disks. The number of Fourier modes is fixed to $m=2 \cdot 200$, i.e. $M=1.6 \times 10^{5}$ degrees of freedom. We compare the solution at each time step with the initial condition on the grid nodes within the considered disk. The worst approximation of the steady-state solution is the commonly used $\rho_{2}$ Padé approximation, whereas $\psi_{0}(r, \theta)=\sqrt{\rho_{4}(r)} \mathrm{e}^{\mathrm{i} \theta}$ turns out to be as accurate as the numerical solution. For this reason, in the following 

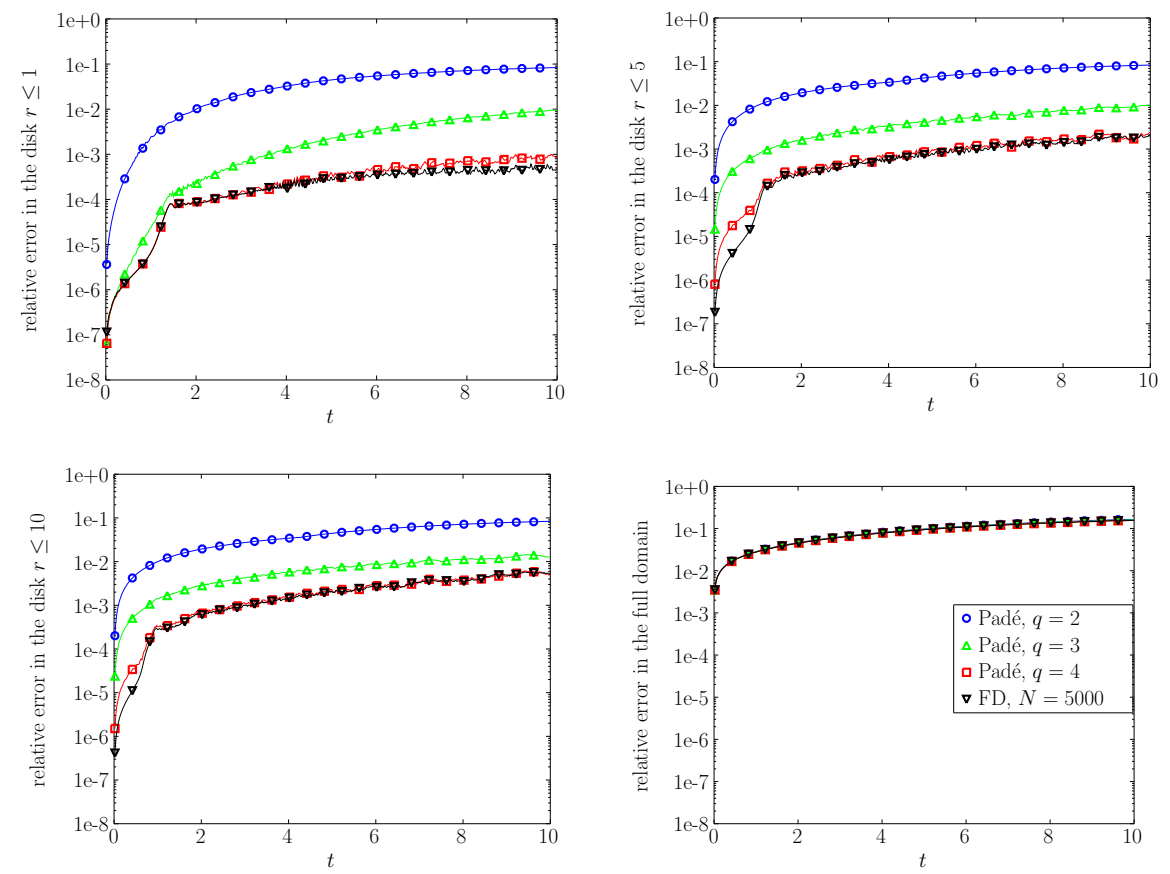

Figure 4: Comparison of the relative error as defined by (17) for different choices of the initial condition, Fourier approach.

experiments we will consider only $\psi_{0}(r, \theta)=\sqrt{\rho_{4}(r)} \mathrm{e}^{\mathrm{i} \theta}$. All curves collapse on each other in the case of the largest disk, meaning that the maximum error occurs at the boundaries, mainly due to the non-periodicity of the solution.

\subsection{Uniform vs. nonuniform finite differences}

We compare the performance of the finite difference approximation on a uniform versus nonuniform grid. The uniform grid has the same step-size $h$ as the grid employed for the Fourier approach and reported in Figure 4. The nonuniform grid is generated by taking into account different constraints. Given the smallest step-size $h_{\min }=h_{1}=0.05$ at the origin (in the vortex core), we linearly increase the step-size according to $h_{i+1}=(1+\delta) h_{i}$ in both $x$ and $y$ and in both positive and negative directions. We choose $\delta$ so as to reach the boundaries exactly, and in order to keep the ratio $K=h_{\max } / h_{1} \approx 10$, where $h_{\max }=\max h_{i}$. The number of points of the nonuniform grid is chosen such that the mean value of $\left\{h_{i}\right\}$ equals the step-size of the uniform grid. These constraints guarantee a reasonable nonuniform grid.

The comparison between the uniform and nonuniform grids is shown in Figure 5. In all cases the initial condition is $\psi_{0}(r, \theta)=\sqrt{\rho_{4}(r)} \mathrm{e}^{\mathrm{i} \theta}$, thus the error for the uniform grid can be compared directly with that in Figure 4 for the case of Padé approximation with $q=4$ (red squares in both Figures). 

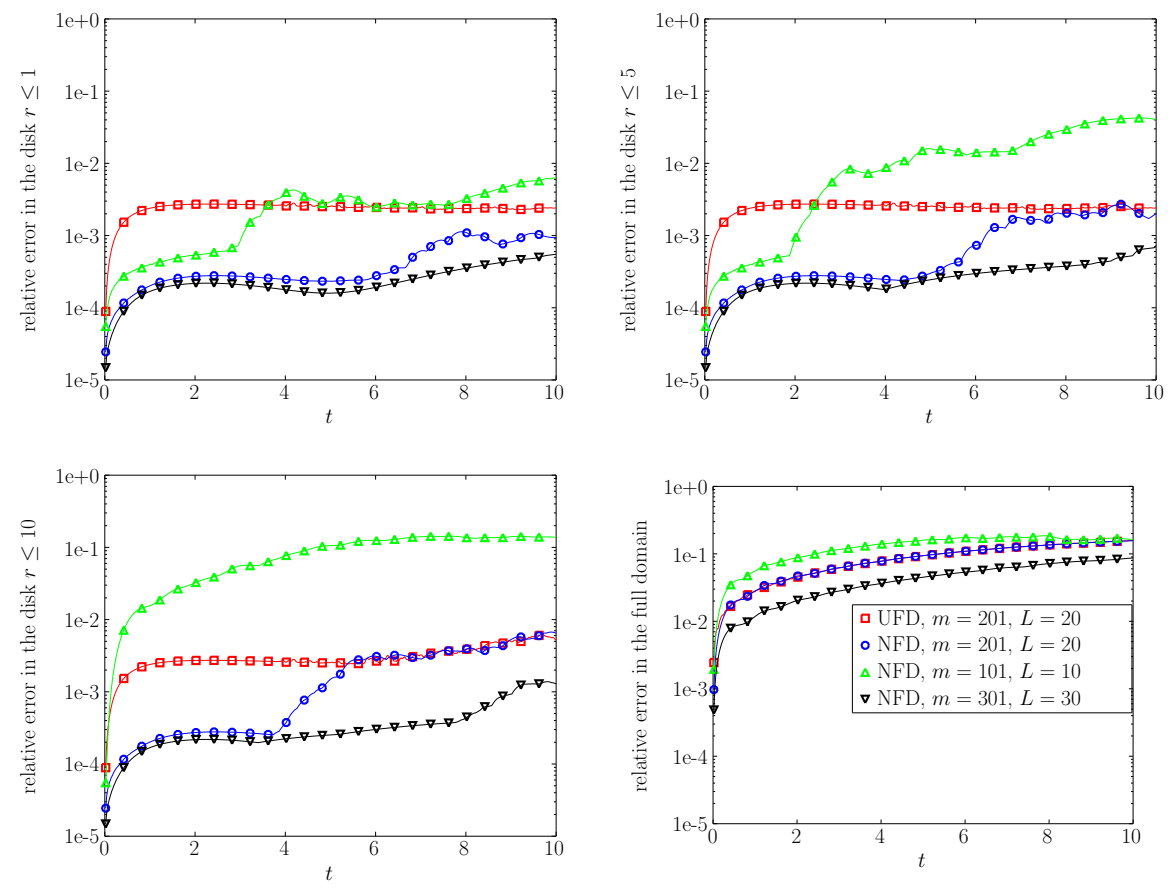

Figure 5: Comparison of the relative error as defined by (17) for central finite differences on uniform (UFD) and nonuniform (NFD) grids.

We first focus on the results with the same number of points and the same boundaries, i.e. red squares and blue circles in Figure 5. The discrete mass variation along time integration is comparable and of order $10^{-13}$. This confirms the conservation of mass also for the case of nonuniform grid, as discussed in Section 3.2 In the nonuniform case the error is roughly one order of magnitude smaller than in the uniform case on small disks and for $t$ not too large, whereas the curve of the nonuniform case tends to jump onto the uniform one after a certain time as the radius of the disk of interest increases. This suggests the idea that the error arises at the boundaries, where $\psi_{0}(r, \theta)$ does not exactly fulfill Neumann boundary conditions.

Motivated by this, we have changed the boundaries from $L=20$ to $L=30$ and $L=10$ to check the dependency of the error on the choice of the truncated domain. In doing so, we have preserved the constraints on the nonuniform grids discussed above, obtaining $M=101^{2}$ degrees of freedom for $L=10$ (upward green triangles) and $M=301^{2}$ degrees of freedom for $L=30$ (downward black triangles). With reference to Figure 5 , the domain bounded at $L=10$ is clearly too small and the error is always very large compared to all the other cases. On the other hand, the curves for $L=20$ (blue circles) and $L=30$ (downward black triangles) behave roughly in the same way up to a certain value of $t$, after 
which the case $L=20$ consistently show larger errors than the case $L=30$. This reinforces the claim that the error arises from the borders.

\subsection{Comparison between Fourier spectral method and nonuniform finite differ- ences}

Now we concentrate on our main goal, which is the comparison between TSSP with Fourier basis function on uniform grids and TSFD on a nonuniform grid that we fix to $h_{\min }=0.05, L=20, m=201$. In order to compare the error defined by (17) for the two methods, we always evaluate the TSSP solution on the nonuniform grid points (spectral solutions can be evaluated everywhere). This set of points has the advantage of being denser in the vortex core, where higher spatial resolution is desirable. Results are reported in Figure 6, where SP stands for spectral and NFD for nonuniform finite differences.
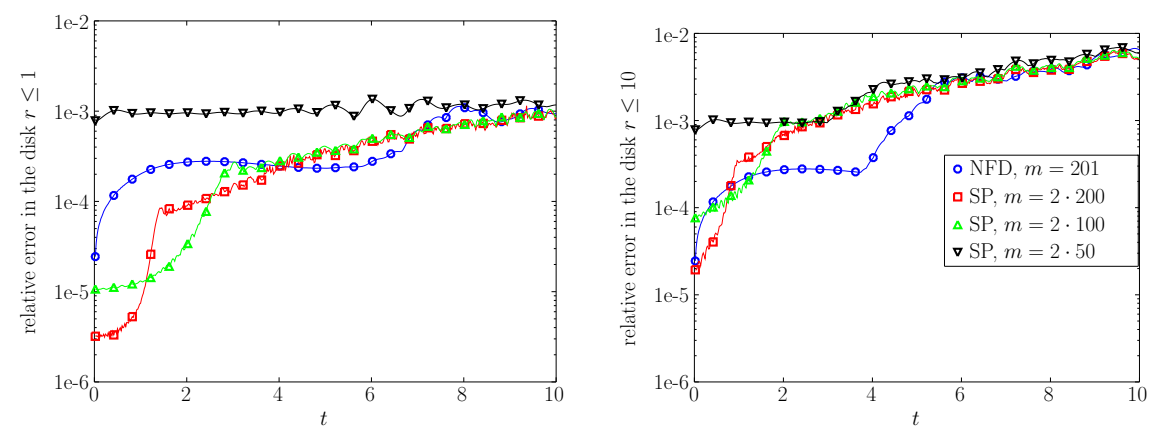

Figure 6: Comparison of the relative error as defined by (17) between nonuniform finite differences (NFD) and spectral Fourier (SP) for different numbers of Fourier modes.

Keeping in mind that the spectral Fourier approach needs mirroring, i.e. the number of modes in each direction must be doubled, we first choose a number of Fourier modes $m=2 \cdot 200$ in each direction to make it equal to the number of points of the reference case for nonuniform finite differences $(m=201)$ in the physical (un-mirrored) domain. The overall behavior of the error for these two cases is comparable: TSSP (red squares) performs better than TSFD (blue circles) for small values of $t$, whereas the opposite happens for intermediate values of $t$. For large $t$ the two curves collapse on each other.

Due to the fact that TSSP needs mirroring, i.e. $M_{\mathrm{TSSP}}=4 M_{\mathrm{TSFD}}$, in Figure 6 we explore also the cases with less Fourier modes, namely $m=2 \cdot 100$ (upward green triangles) and $m=2.50$ (downward black triangles). As observed for the case $m=2 \cdot 200$, in the long term all curves seem to provide similar errors, regardless of the disk radius. On the other hand, for small values of $t$, the number of degrees of freedom plays a rôle in that a larger number of Fourier modes ensures smaller errors.

It is important to keep in mind that, for what seen in Figure 3, the TSSP Fourier approach does not retain the spectral accuracy because of the singular nature of the solution at the origin and the lack of periodicity at the boundaries. 
As a final remark, we observe that the error of the Fourier solution computed on its own uniform grid, reported in Figure 4 with red squares, is smaller than the error of the Fourier solution evaluated on the nonuniform grid, reported in Figure 6 with red squares.

\subsection{Maximum resolution of Fourier spectral method}

As expected, from Figure 6 we have seen that the smaller the number of Fourier modes, the larger the relative error with respect to the initial condition.
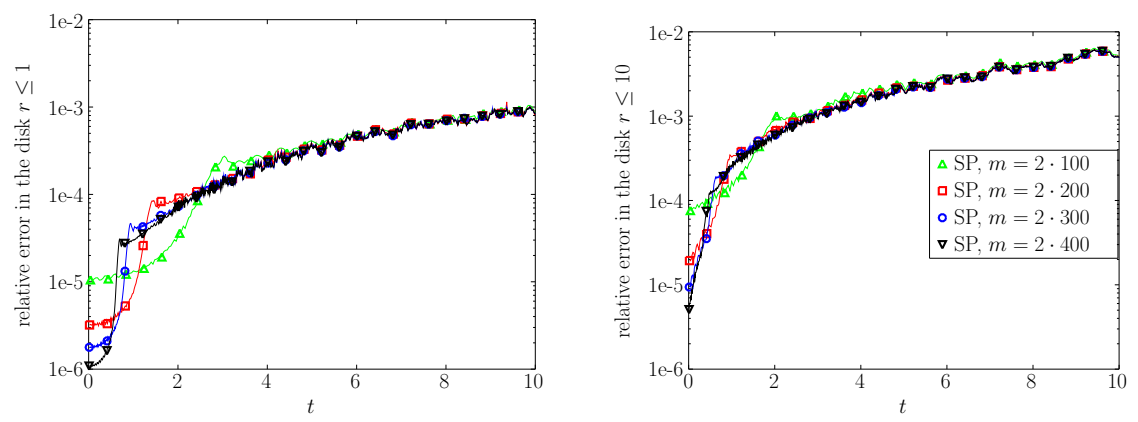

Figure 7: Comparison of the relative error as defined by (17) for increasing number of Fourier modes.

We wish to check if there exists an upper limit to the maximum resolution of Fourier spectral method. For doing so, we increase the number of Fourier modes and, proportionally, the number of time steps as suggested in [27]. Results are shown in Figure 7 We observe high accuracy in the core (see smaller disk, left plot) for small values of $t$, immediately followed by saturation. In a larger disk (right plot), saturation kicks in almost immediately. The errors reported in Figure 7 suggest that $m=2 \cdot 200$ is a reasonable value of Fourier modes for the preservation of a two-dimensional quantum vortex.

\subsection{Fourier evaluation on nonuniform grids}

As explained in the Introduction, our motivation to explore the nonuniform finite difference approach is based on the need, for the study of vortex reconnections [10], of high local spatial resolution and, possibly, accuracy.

Instead of increasing the number of Fourier modes so as to reach higher global spatial resolution, one can resort to a TSSP method with a reasonable number of modes (considering that mirroring is needed) and then evaluate the TSSP solution on a nonuniform grid, with denser points where they are needed. In Figure 8 we compare the reasonable case $m=2 \cdot 100$, for which the number of modes is relatively small, but not too small, with different nonuniform grids. We notice that the numerical integration itself is carried out only once and the Fourier coefficients of the solution are stored at each time step. The evaluation at the grid points is performed afterward, in the post-processing stage, as many 

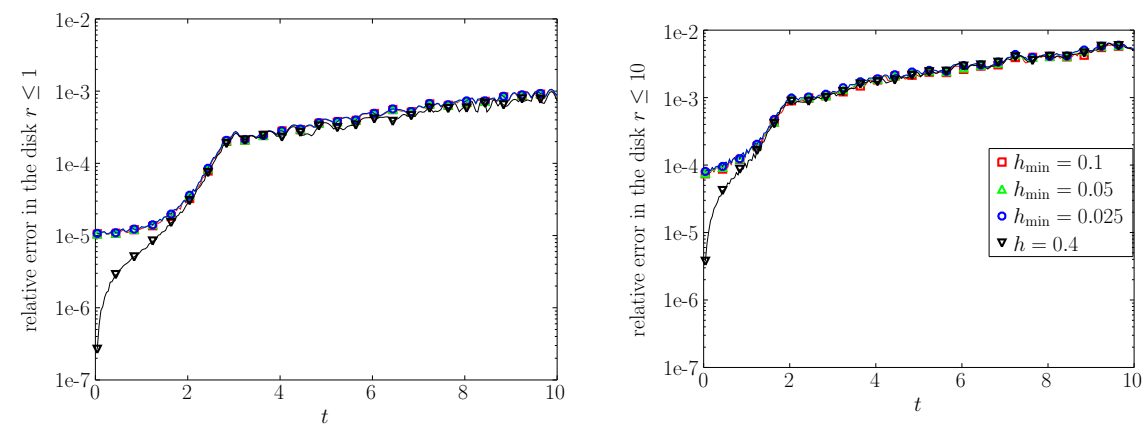

Figure 8: Comparison of the relative error as defined by (17) for different evaluations of the Fourier solution $(m=2 \cdot 100)$ at nonuniform grids.

times as desired. Moreover, tools like the Nonuniform Fast Fourier Transform (NFFT, see [28]) can be employed for the fast evaluation of trigonometric polynomials at arbitrary point sets. The constant spatial step-size of the TSSP method is $h=0.4$, whereas $h_{\min }$ stands for the minimum value of the step-size, in the proximity of the origin, for the nonuniform grids. As seen before, there is a substantial difference in the error only for $t<2$, whereas for larger values of $t$ evaluating the Fourier solution on a nonuniform grid does not worsen the solution. It is important to note that vortex reconnections, usually, require a dynamics that takes a time of at least $t=10$. Evaluating a TSSP solution on a locally refined grid is, thus, a very promising approach to study quantum vortex reconnections.

\section{Conclusions}

After deriving a new accurate Padé approximation for the density distribution of a two-dimensional steady-state vortex, we have used it as the initial condition for the Gross-Pitaevskii equation to test the performance of the time-splitting Fourier method. Although it cannot retain its classical spectral accuracy in space, being as accurate as low-order finite difference on nonuniform grids, it preserves quite well the steady-state solution, especially in the neighborhood of the singularity. The advantage of a post-processing evaluation on arbitrary points makes this approach suited for applications where local high resolution is required.

\section{References}

\section{References}

[1] W. F. Vinen, An introduction to quantum turbulence, Phil. Trans. R. Soc. A 366 (1877) (2008) 2925-2933. 
[2] M. S. Paoletti, D. P. Lathrop, Quantum turbulence, Ann. Rev. Cond. Mat. Phys. 2 (2011) 213-234.

[3] C. F. Barenghi, L. Skrbek, K. R. Sreenivasan, Introduction to quantum turbulence, Proc. Natl. Acad. Sci. USA 111 (1) (2014) 4647-4652.

[4] U. Frisch, Turbulence, Cambridge University Press, Cambridge, England, 1995.

[5] S. B. Pope, Turbulent Flows, Cambridge University Press, Cambridge, England, 200.

[6] F. Hussain, K. Duraisamy, Mechanics of viscous vortex reconnection, Phys. Fluids 23 (2011) 021701(4).

[7] S. Zuccher, M. Caliari, A. W. Baggaley, C. F. Barenghi, Quantum vortex reconnections, Phys. Fluids 24 (125108) (2012) 1-21.

[8] L. P. Pitaevskii, Vortex lines in an imperfect Bose gas, Sov. Phys. JETP 13 (1961) 451-54.

[9] E. P. Gross, Hydrodynamics of a superfluid condensate, J. Math. Phys. 4 (1963) 195-207.

[10] S. Zuccher, R. L. Ricca, Helicity conservation under quantum reconnection of vortex rings, Phys. Rev. E 92 (6) (2015) 061001.

[11] L. Kondaurova, V. L'vov, A. Pomyalov, I. Procaccia, Structure of a quantum vortex tangle in ${ }^{4} \mathrm{He}$ counterflow turbulence, Phys. Rev. B 89 (2014) 014502 .

[12] M. D. Bustamante, R. M. Kerr, 3D Euler about a 2D symmetry plane, Physica D 237 (2008) 1912-1920.

[13] R. Hänninen, A. W. Baggaley, Vortex filament method as a tool for computational visualization of quantum turbulence, Proc. Natl. Acad. Sci. USA 111 (1) (2014) 4667-4674.

[14] J. Koplik, H. Levine, Vortex Reconnection in Superfluid Helium, Phys. Rev. Lett. 71 (9) (1993) 1375-1379.

[15] A. J. Allen, S. Zuccher, M. Caliari, N. Proukakis, N. G. Parker, C. F. Barenghi, Vortex reconnections in atomic condensates at finite temperature, Phis. Rev. A 90 (2014) 013601.

[16] M. W. Scheeler, D. Kleckner, D. Proment, G. L. Kindlmann, W. T. M. Irvine, Helicity conservation by flow across scales in reconnecting vortex links and knots, Proc. Natl. Acad. Sci. USA 111 (43) (2014) 15350-15355.

[17] N. G. Berloff, Padé approximations of solitary wave solutions of the GrossPitaevskii equation, J. Phys. A: Math. Gen. 37 (2004) 1617-1632. 
[18] S. Nazarenko, R. West, Analytical solution for nonlinear Schrödinger vortex reconnection, J. Low Temp. Phys. 132 (1) (2003) 1-10.

[19] D. P. Meichle, C. Rorai, M. E. Fisher, D. P. Lathrop, Quantized vortex reconnection: Fixed points and initial conditions, Phys. Rev. B 86 (1) (2012) 014509.

[20] W. Bao, Y. Cai, Mathematical theory and numerical methods for BoseEinstein condensation, Kinet. Relat. Models 6 (1) (2013) 1-135.

[21] M. Thalhammer, M. Caliari, C. Neuhauser, High-order time-splitting Hermite and Fourier spectral methods, J. Comput. Phys. 228 (3) (2009) 822832.

[22] V. Gradinaru, Strang splitting for the time-dependent Schrödinger equation on sparse grids, SIAM J. Numer. Anal. 46 (1) (2007) 103-123.

[23] M. Thalhammer, J. Abhau, A numerical study of adaptive space and time discretisations for Gross-Pitaevskii equations, J. Comput. Physics 231 (20) (2012) 6665-6681.

[24] J. H. Ferziger, M. Perić, Computational Methods for Fluid Dynamics, 3rd Edition, Springer, 2002.

[25] S.-C. T. Choi, Minimal residual methods for complex symmetric, skew symmetric, and skew hermitian systems, Tech. Rep. ANL/MCS-P3028-0812, Computation Institute, University of Chicago, Chicago, Illinois (2013).

[26] M. Caliari, P. Kandolf, A. Ostermann, S. Rainer, Comparison of software for computing the action of the matrix exponential, BIT 54 (1) (2014) $113-128$.

[27] W. Bao, S. Jin, P. A. Markowich, On time-splitting spectral approximations for the Schrödinger equation in the semiclassical regime, J. Comput. Phys. 175 (2) (2002) 487-524.

[28] J. Keiner, S. Kunis, D. Potts, Using NFFT 3-A Software Library for Various Nonequispaced Fast Fourier Transforms, ACM Trans. Math. Software 36 (4) (2009) 19:1-19:30.

\section{Appendix A. Detailed derivation of Padé approximations}

The case $q=2$. The coefficients of this expansion are already known, however it is instructive to proceed with their derivation in order to understand how it works. We have to compute 3 coefficients, $a_{1}, b_{1}$ and $a_{2}$, therefore we can use only 3 equations. These equations are obtained by nullifying, respectively, the coefficients of the terms $r^{2}, r^{4}$ and $r^{6}$ in the numerator $N_{2}(r)$ (lower-order powers of $r^{2 k}$ ). By nullifying the coefficient of $r^{2}$, we get

$$
-4 a_{1}^{2} b_{1}+4 a_{1} a_{2}+a_{1}^{2}=0
$$




\begin{tabular}{c|cc} 
& $q=2$ & $q=3$ \\
\hline$a_{1}$ & $\frac{11}{32}$ & 0.34003812123694735361 \\
$b_{1}$ & $\frac{5-32 a_{1}}{48-192 a_{1}}$ & $\frac{2304 a_{1}^{3}+656 a_{1}^{2}-421 a_{1}-28}{7680 a_{1}^{2}-1680 a_{1}-330}$ \\
$a_{2}$ & $a_{1}\left(b_{1}-\frac{1}{4}\right)$ & $a_{1}\left(b_{1}-\frac{1}{4}\right)$ \\
$b_{2}$ & & $\frac{768 a_{1} b_{1}-120 b_{1}-384 a_{1}^{2}+8 a_{1}+7}{4608 a_{1}-1152}$ \\
$a_{3}$ & & $\frac{a_{1}\left(192 b_{2}-48 b_{1}+16 a_{1}+5\right)}{192}$
\end{tabular}

Table A.1: Coefficients of Padé approximations $\rho_{2}$ and $\rho_{3}$.

from which $a_{2}=a_{1}\left(b_{1}-\frac{1}{4}\right)$. By nullifying the coefficient of $r^{4}$, and replacing $a_{2}$ with the expression above, we get

$$
a_{1}^{2}\left(192 a_{1} b_{1}-48 b_{1}-32 a_{1}+5\right)=0,
$$

which gives $b_{1}=\frac{5-32 a_{1}}{48-192 a_{1}}$. If we now nullify the coefficient of $r^{6}$ and replace $a_{2}$ with $a_{1}\left(b_{1}-\frac{1}{4}\right)$ and $b_{1}$ with $\frac{5-32 a_{1}}{48-192 a_{1}}$, we get the following equation

$$
a_{1}^{2}\left(8 a_{1}+1\right)\left(32 a_{1}-11\right)=0 .
$$

Clearly, $a_{1}=0$ is not acceptable, nor is $a_{1}=-\frac{1}{8}$. The only acceptable value is $a_{1}=\frac{11}{32}$. As we mentioned before, equation (8) cannot be satisfied exactly, however, an a posteriori evaluation reveals that the remaining coefficients of $r^{2 k}$ are smaller than $1.5 \times 10^{-4}$ and monotonically decreasing with $k$.

The case $q=3$. Since we have to compute 5 coefficients we need 5 equations, which are obtained by imposing that the coefficients of the terms $r^{2}, r^{4}, r^{6}, r^{8}$ and $r^{10}$ must be zero. By nullifying the coefficient of $r^{2}$ we still get the same equation as for $q=2,-4 a_{1}^{2} b_{1}+4 a_{1} a_{2}+a_{1}^{2}=0$, from which $a_{2}=a_{1}\left(b_{1}-\frac{1}{4}\right)$. By nullifying the coefficient of $r^{4}$, and replacing $a_{2}$ with the expression above, we get

$$
192 a_{1} b_{2}-48 a_{1} b_{1}-192 a_{3}+16 a_{1}^{2}+5 a_{1}=0,
$$

which is easy to solve for $a_{3}$ leading to

$$
a_{3}=\frac{a_{1}\left(192 b_{2}-48 b_{1}+16 a_{1}+5\right)}{192} .
$$

Now we collect terms in $r^{6}$ and impose its coefficient to be zero. In this equation we replace $a_{2}$ and $a_{3}$ with the expressions derived above and get the equation

$$
4608 a_{1} b_{2}-1152 b_{2}-768 a_{1} b_{1}+120 b_{1}+384 a_{1}^{2}-8 a_{1}-7=0,
$$




\begin{tabular}{c|c} 
& $q=4$ \\
\hline$a_{1}$ & 0.34010790700196714760 \\
$b_{1}$ & $\frac{2304 a_{1}^{3}+656 a_{1}^{2}-421 a_{1}-28}{7680 a_{1}^{2}-1680 a_{1}-330}$ \\
$a_{2}$ & $a_{1}\left(b_{1}-\frac{1}{4}\right)$ \\
$b_{2}$ & $\frac{\left(737280 a_{1}^{3}+209920 a_{1}^{2}-134720 a_{1}-8960\right) b_{1}-364544 a_{1}^{3}+70144 a_{1}^{2}+18256 a_{1}+393}{2457600 a_{1}^{2}-537600 a_{1}-105600}$ \\
$a_{3}$ & $\frac{a_{1}\left(192 b_{2}-48 b_{1}+16 a_{1}+5\right)}{192}$ \\
$b_{3}$ & $\frac{\left(61440 a_{1}-9600\right) b_{2}+\left(-30720 a_{1}^{2}+640 a_{1}+560\right) b_{1}+8448 a_{1}^{2}-1056 a_{1}-21}{368640 a_{1}-92160}$ \\
$a_{4}$ & $\frac{4608 a_{1} b_{3}-1152 a_{1} b_{2}+\left(384 a_{1}^{2}+120 a_{1}\right) b_{1}-128 a_{1}^{2}-7 a_{1}}{4608}$
\end{tabular}

Table A.2: Coefficients of Padé approximation $\rho_{4}$.

which we solve for $b_{2}$ :

$$
b_{2}=\frac{768 a_{1} b_{1}-120 b_{1}-384 a_{1}^{2}+8 a_{1}+7}{4608 a_{1}-1152} .
$$

Then we nullify the coefficient of $r^{8}$, substitute all previously found $a_{2}, a_{3}$ and $b_{2}$, getting the equation

$$
7680 a_{1}^{2} b_{1}-1680 a_{1} b_{1}-330 b_{1}-2304 a_{1}^{3}-656 a_{1}^{2}+421 a_{1}+28=0,
$$

which gives

$$
b_{1}=\frac{2304 a_{1}^{3}+656 a_{1}^{2}-421 a_{1}-28}{7680 a_{1}^{2}-1680 a_{1}-330} .
$$

Finally, we nullify the coefficient of $r^{10}$, substitute $a_{2}, a_{3}, b_{2}$ and $b_{1}$, and get the equation for $a_{1}$

$$
a_{1}^{2}\left(21233664 a_{1}^{5}-9732096 a_{1}^{4}-62464 a_{1}^{3}+137856 a_{1}^{2}+62772 a_{1}-1247\right)=0 .
$$

This equation must be solved numerically and leads to many real solutions. However the only value that reproduces a physical behavior of $\rho_{3}(r)$ for $r \rightarrow 0$ is $a_{1}=0.34003812123694735361$. It is possible to compute the first derivative and verify that $\rho_{3}^{\prime}(r)>0$ for all $r>0$. In other words, $\rho_{3}$ is a physical, monotonically increasing, approximation of the density due to a two-dimensional quantum vortex. Again, the coefficients of $r^{2 k}$ that are not zero are, indeed, smaller than $4.0 \times 10^{-4}$ and monotonically decreasing with $k$.

The case $q=4$. Now we have 7 coefficients to compute, therefore we need 7 equations, i.e. we need to nullify the coefficients of $r^{2 k}$ for $k=1, \ldots, 7$. 
By canceling the term $r^{2}$ and solving for $a_{2}$ we get the usual expression $a_{2}=$ $a_{1}\left(b_{1}-\frac{1}{4}\right)$. By nullifying the term $r^{4}$, substituting $a_{2}$ and solving for $a_{3}$ we get $a_{3}=\frac{a_{1}\left(192 b_{2}-48 b_{1}+16 a_{1}+5\right)}{192}$, which is the same expression obtained for $\rho_{3}$. By canceling the term $r^{6}$, substituting $a_{2}$ and $a_{3}$ as found, and solving for $a_{4}$ we get

$$
a_{4}=\frac{4608 a_{1} b_{3}-1152 a_{1} b_{2}+\left(384 a_{1}^{2}+120 a_{1}\right) b_{1}-128 a_{1}^{2}-7 a_{1}}{4608} .
$$

By canceling the term $r^{8}$, substituting $a_{2}, a_{3}, a_{4}$ and solving for $b_{3}$ we get

$$
b_{3}=\frac{\left(61440 a_{1}-9600\right) b_{2}+\left(-30720 a_{1}^{2}+640 a_{1}+560\right) b_{1}+8448 a_{1}^{2}-1056 a_{1}-21}{368640 a_{1}-92160} .
$$

By canceling the term $r^{10}$, substituting $a_{2}, a_{3}, a_{4}$ and $b_{3}$, and solving for $b_{2}$ we get

$$
b_{2}=\frac{\left(737280 a_{1}^{3}+209920 a_{1}^{2}-134720 a_{1}-8960\right) b_{1}-364544 a_{1}^{3}+70144 a_{1}^{2}+18256 a_{1}+393}{2457600 a_{1}^{2}-537600 a_{1}-105600} .
$$

By canceling the term $r^{12}$, substituting all known $a_{j}, b_{3}, b_{2}$, and solving for $b_{1}$ we get

$$
b_{1}=\frac{722731008 a_{1}^{5}-326467584 a_{1}^{4}-13427712 a_{1}^{3}+11551104 a_{1}^{2}+834006 a_{1}-12183}{2972712960 a_{1}^{5}-1362493440 a_{1}^{4}-8744960 a_{1}^{3}+19299840 a_{1}^{2}+8788080 a_{1}-174580}
$$

Finally by canceling the term $r^{14}$, substituting all $a_{j}, b_{k}$ previously found, we get an equation for $a_{1}$

$$
\begin{aligned}
& 1292033536819200 a_{1}^{8}-2530164294549504 a_{1}^{7}+1853440540016640 a_{1}^{6}- \\
& 642522859438080 a_{1}^{5}+107808283328512 a_{1}^{4}-8028170208256 a_{1}^{3}+ \\
& 248539665024 a_{1}^{2}+1297120628 a_{1}+9325957=0 .
\end{aligned}
$$

This equation has many real solutions, which can be determined numerically. However, the only value that leads to a physically acceptable $\rho_{4}(r)$ for $r \rightarrow 0$ is $a_{1}=0.34010790700196714760$. After computing all other coefficients and the first derivative, it is straighforward to verify that $\rho_{4}^{\prime}(r)>0$ for all $r>0$, i.e. $\rho_{4}$ is a physical, monotonically increasing, approximation of the density for a two-dimensional quantum vortex. As observed before, the coefficients of $r^{2 k}$ that are not zero are smaller than $1.9 \times 10^{-11}$ and monotonically decreasing with $k$. 